\title{
Optimization of Operation Sequencing in CAPP Using Superhybrid Genetic Algorithms-Simulated Annealing Technique
}

\author{
G. Nallakumarasamy, ${ }^{1}$ PSS. Srinivasan, ${ }^{2}$ K. Venkatesh Raja, ${ }^{1}$ and R. Malayalamurthi ${ }^{3}$ \\ ${ }^{1}$ Department of Automobile Engineering, K.S.R. College of Engineering, Tamil Nadu, Tiruchengode 637215, India \\ ${ }^{2}$ Department of Mechanical Engineering, Knowledge Institute of Technology, Tamil Nadu, Salem 637504, India \\ ${ }^{3}$ Department of Mechanical Engineering, Government College of Engineering, Tamil Nadu, Salem 636011, India
}

Correspondence should be addressed to K. Venkatesh Raja, kvenkateshraja@hotmail.com

Received 28 March 2011; Accepted 5 May 2011

Academic Editors: G. A. Britton and J.-L. Marcelin

Copyright (C) 2011 G. Nallakumarasamy et al. This is an open access article distributed under the Creative Commons Attribution License, which permits unrestricted use, distribution, and reproduction in any medium, provided the original work is properly cited.

Computer-aided process planning (CAPP) is an important interface between computer-aided design (CAD) and computer-aided manufacturing (CAM) in computer-integrated manufacturing environment. A problem in traditional CAPP system is that the multiple planning tasks are treated in a linear approach. This leads to an overconstrained overall solution space, and the final solution is normally far from optimal or even nonfeasible. A single sequence of operations may not be the best for all the situations in a changing production environment with multiple objectives such as minimizing number of setups, maximizing machine utilization, and minimizing number of tool changes. In general, the problem has combinatorial characteristics and complex precedence relations, which makes the problem more difficult to solve. The main contribution of this work is to develop an intelligent CAPP system for shop-floor use that can be used by an average operator and to produce globally optimized results. In this paper, the feasible sequences of operations are generated based on the precedence cost matrix (PCM) and reward-penalty matrix (REPMAX) using superhybrid genetic algorithms-simulated annealing technique (S-GENSAT), a hybrid metaheuristic. Also, solution space reduction methodology based on PCM and REPMAX upgrades the procedure to superhybridization. In this work, a number of benchmark case studies are considered to demonstrate the feasibility and robustness of the proposed super-hybrid algorithm. This algorithm performs well on all the test problems, exceeding or matching the solution quality of the results reported in the literature. The main contribution of this work focuses on reducing the optimal cost with a lesser computational time along with generation of more alternate optimal feasible sequences. Also, the proposed S-GENSAT integrates solution space reduction, hybridization, trapping out of local minima, robustness, and convergence; it consistently outperformed both a conventional genetic algorithm and a conventional simulated annealing algorithm.

\section{Introduction}

This section presents a brief overview of the CAPP and importance of sequencing, a short description of the complexity of this class of problem, and the need for global search techniques to efficiently solve it.

Process planning is defined as the activity of deciding which manufacturing processes and machines should be used to perform the various operations necessary to produce a component, and the sequence that the processes should follow. Alternatively, process planning is the systematic determination of the detailed methods by which parts can be manufactured from raw material to finished product.
The process planning activity includes interpretation of design data, selection and sequencing of operation to manufacture the part, selection of machines and cutting tools, determination of cutting parameters, choice of jigs and fixtures, and calculation of machining times and costs. CAPP is considered as the key technology for computeraided design/manufacturing (CAD/CAM) integration. There are two basic approaches to CAPP: variant and generative. The variant approach uses group technology concepts like classification and coding systems to select a generic process plan from the existing master process plans developed for each part family, and edits to suit the requirement of the part. In the generative approach, a process plan is generated 
by synthesizing the part data with the information from manufacturing databases and decision rules. In the recent years, process planning has received significant attention from researchers, and numerous approaches have been proposed in order to obtain optimal or near-optimal solutions of this intractable problem. Unfortunately, the available machining resources in the job shop, geometrical as well as technological requirements of the part, and precedence relationships among all the operations make the conducting of operations selection and operations sequencing simultaneously a combinatorial optimization problem.

Stochastic global search algorithms such as genetic algorithms are used to attack difficult combinatorial optimization problems. However, genetic algorithms suffer from the lack of a convergence proof. It is difficult to establish reliable algorithm braking criteria without extensive knowledge of the solution space. The proposed hybrid genetic algorithm combines a genetic algorithm with simulated annealing in order to overcome the algorithm convergence problem. The hybrid algorithm was used successfully to solve an operation sequencing problem.

The two major tasks in process planning are: operation selection and operation sequencing, respectively. The operation selection is based on the form-feature geometry, its technological requirements, and mapping these specifications to the appropriate operation or series of operations [1]. Even though there subsists a huge number of CAPP systems in the literature. However, a very few of them have intended to provide globally-optimized operation sequences. To determine the optimal sequence, various classical techniques like branch and bound methods, linear programming, and dynamics programming have been extensively discussed in detail [2-4], and demonstrated a strategy for CAPP in the single-machine case using a feature precedence graph to represent the relative costs of set-up changes required for any two consecutive operations. The least cost optimal process route was identified by Hamilton path of precedence graph. Further, they compared Latin multiplication method with Kernighan heuristic for randomly generated alternative plans. Usher and Bowden [5] presented an approach for operation sequence coding that permits the application of genetic algorithm (GA) for quick determination of optimal, or near-optimal, operation sequences for parts having varying complexity.

As the operations sequencing problem involves various interdependent constraints, it is very difficult to formulate and solve this problem using classical techniques alone. Recently, most works applied metaheuristics for solving process planning problems. Bhaskara Reddy et al. [6] applied genetic algorithms to generate the optimal sequence of manufacturing operations. The feasible sequences are generated from the feature precedence relationship based on the precedence and geometrical tolerance constraints. Turkey Dereli and Filiz [7] emphasized the optimization of all process parameters of the CAPP systems. He also presented the methodologies used for the development of GA-based systems responsible for optimization of sequence of operations, optimization of ATCindex positions, and optimization of cutting parameters. Lee et al. [8] focused on the operation sequencing problem with the objective of minimizing the sum of machine setup and tool change costs.

Zhang et al. [9] developed an algorithm based on GA to find the optimal solution. Based on their studies, they have concluded that the approach was more realistic and possible to find a global optimal process plan. Foerster and Wäscher [10] used simulated annealing for order spread minimization in sequencing cutting patterns (OSMP) which is classified as NP-complete problem and it can be considered as a generalized Travelling-Salesman Problem (TSP). Li et al. [11] investigated the application of constrained-based tabu search approach for optimization of process plans. Further it was investigated by Krishna and Rac [12] using Ant colony algorithm (ACA) and found that the computational time has considerably reduced. Guo et al. [13] applied particle swarm optimization (PSO) for operation sequencing problem, and concluded that there is still potential for further improvement in computation efficiency and optimality if introducing new operators and characteristics of other algorithms. Furthermore, Salehi et al. [14] again applied genetic algorithms to generate the optimal sequence of manufacturing operations in preliminary and detailed planning. Nallakumarasamy et al. [15] investigated the operation sequencing problem using simulated annealing technique (SAT) and concluded that SAT is superior to GA and ACA. Wang et al. [16] applied hybrid particle swarm optimization for process planning problem and suggested that the algorithm has shown the capability for attaining a good quality of solution. Ganesh and Punniyamoorthy [17] developed a hybrid GA SA algorithm and found that the algorithm performs better. Among these heuristic methods, Genetic Algorithms and simulated annealing represent powerful combinatorial optimization methods with complementary strength and weakness and are naturally motivated.

However, to the author knowledge, application of a hybrid heuristic algorithm using GA and SAT incorporating solution space reduction technique (SSRT) has not been addressed. In this work, an attempt has been made for developing an efficient search technique to obtain feasible optimal solution with a minimal computational time. Evolutionary algorithms, which mimic living organisms in achieving optimal survival solutions, can often outperform conventional optimization methods. In the past two decades, GA has been widely applied for solving complex manufacturing problems. GA operates on the whole population, and the search process may be trapped in local optima, while SA possess the capability of jumping out of local minima and proceeds towards global minima. Essence of both the algorithms are merged together to activate the superhybrid algorithm.

\section{Modeling of Process Planning Problem}

Computer aided process planning encompasses the following phases. First, the part drawing is evaluated for recognizing the form features to be machined, along with details of geometric conditions and technological data such as tolerance and surface finish requirements. This is followed by 


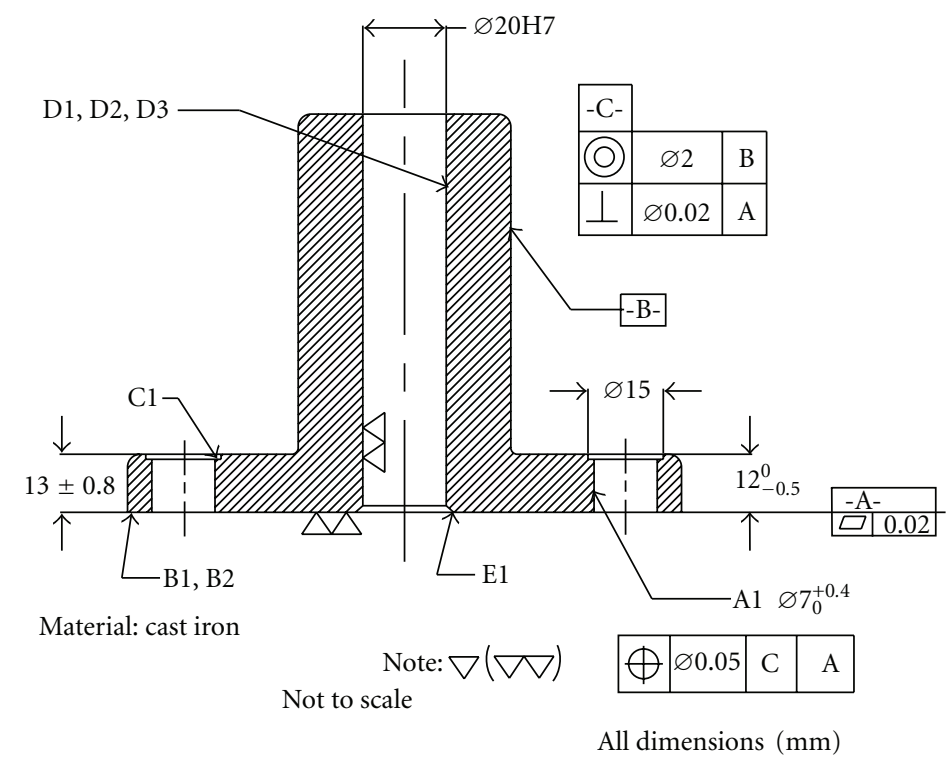

Figure 1: Part drawing for case study 1 [6].

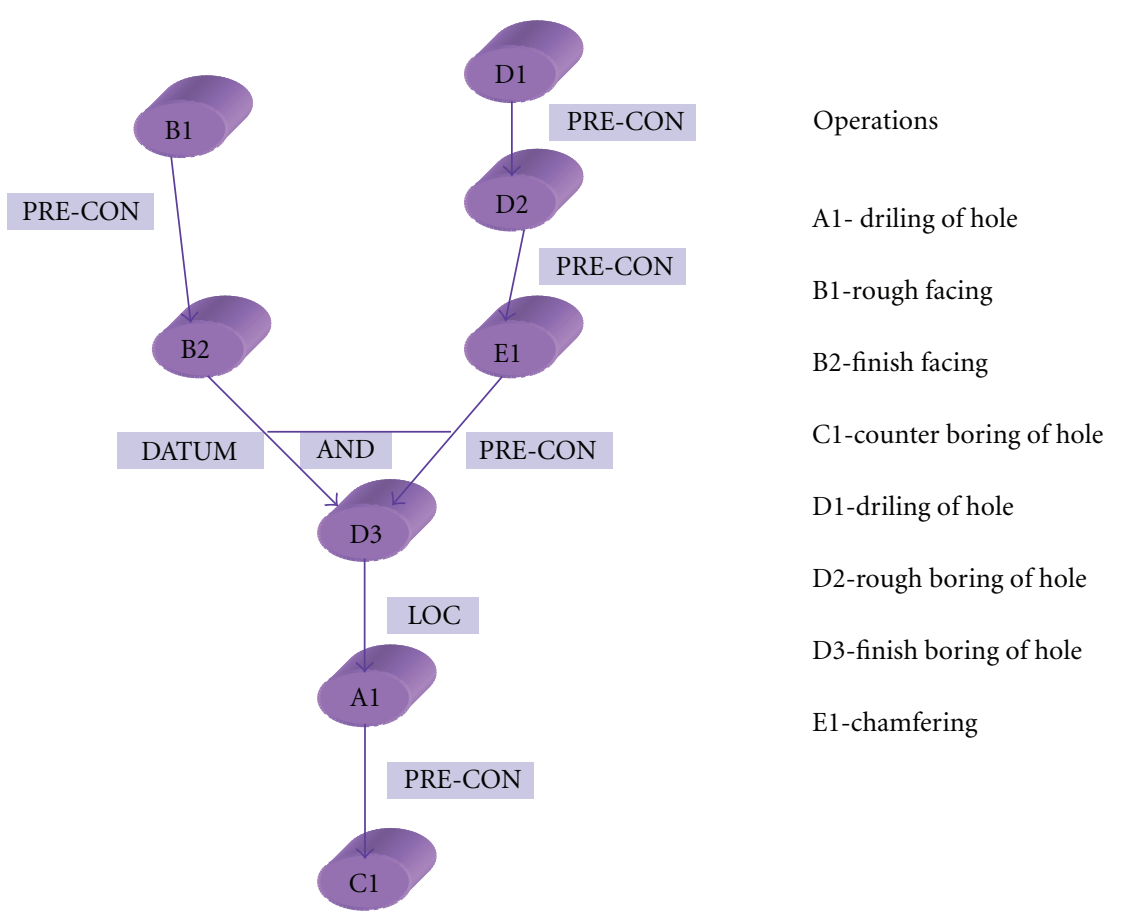

Figure 2: Operations precedence graph for component shown in Figure 1 [6].

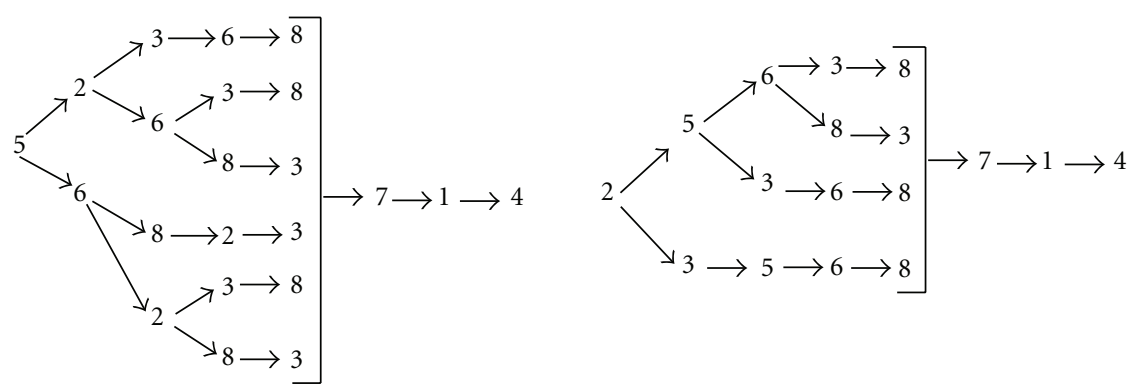

FigURE 3: SSRT-based feasible sequences. 
Table 1: Precedence cost matrix for component shown in Figure 1 [6].

\begin{tabular}{|c|c|c|c|c|c|c|c|c|c|}
\hline \multicolumn{10}{|c|}{ Succeeding operations $\rightarrow$} \\
\hline & & 1 & 2 & 3 & 4 & 5 & 6 & 7 & 8 \\
\hline (A1) & 1 & - & 100 & 100 & 1 & 100 & 100 & 100 & 100 \\
\hline (B1) & 2 & 11 & - & 0 & 100 & 1 & 100 & 100 & 100 \\
\hline (B2) & 3 & 11 & 100 & - & 100 & 1 & 100 & 1 & 1 \\
\hline$(\mathrm{C} 1)$ & 4 & 100 & 100 & 100 & - & 100 & 100 & 100 & 100 \\
\hline (D1) & 5 & 11 & 1 & 100 & 100 & - & 0 & 100 & 100 \\
\hline (D2) & 6 & 11 & 1 & 100 & 100 & 100 & - & 100 & 100 \\
\hline (D3) & 7 & 11 & 100 & 100 & 100 & 100 & 100 & - & 100 \\
\hline (E1) & 8 & 11 & 1 & 100 & 100 & 100 & 100 & 1 & - \\
\hline
\end{tabular}

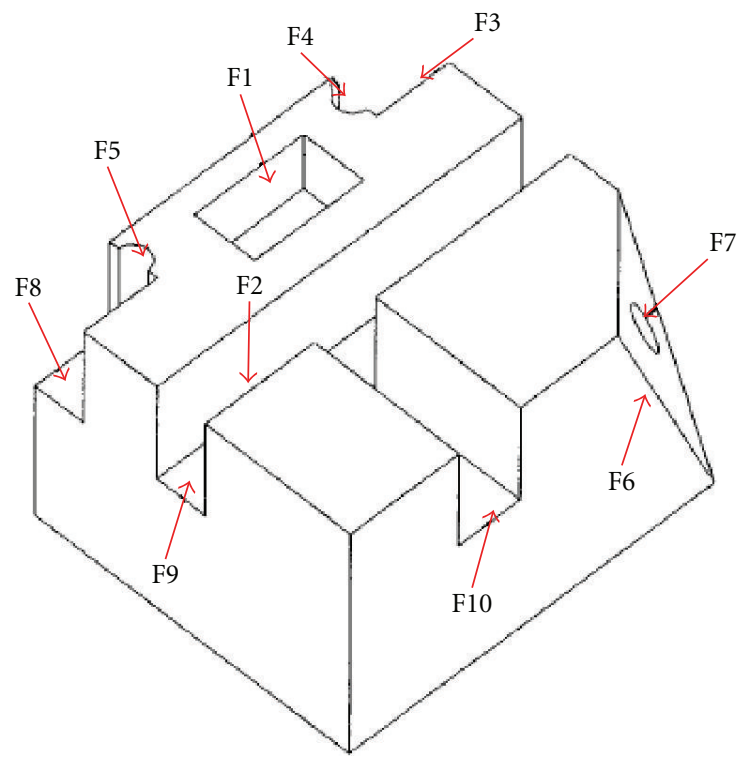

FIgURe 4: Part drawing for case study 2 [7].

TABLE 2: Optimal cost for part shown in Figure 1 Using SSRT.

\begin{tabular}{lcc}
\hline S. No & Sequence & Cost \\
\hline 1 & $5-2-3-6-8-7-1-4$ & 214 \\
2 & $5-2-6-3-8-7-1-4$ & 214 \\
3 & $5-2-6-8-3-7-1-4$ & 314 \\
4 & $5-6-8-2-3-7-1-4$ & 114 \\
5 & $5-6-2-3-8-7-1-4$ & 15 \\
6 & $5-6-2-8-3-7-1-4$ & 214 \\
7 & $2-5-6-3-8-7-1-4$ & 115 \\
8 & $2-5-6-8-3-7-1-4$ & 214 \\
9 & $2-5-3-6-8-7-1-4$ & 314 \\
10 & $2-3-5-6-8-7-1-4$ & 114 \\
\hline
\end{tabular}

the selection of machining operations, machine tools, cutting tools, and cutting parameters required to generate each of the form features. The precedence association among the machining operations is acquired, considering various feasibility constraints, and is discussed in detail in the following section. A relative cost matrix is generated using the feature precedence and their attribute values, such as machine change, tool change, set-up change, and machining parameter change based on the knowledge of various manufacturing processes and capabilities of the machine tools in the shop floor. The generation of feasible plans has the structure of the well-known Travelling Salesman Problem (TSP) in graph theory literature.

2.1. Operation Sequencing. The nature of operation sequence generation is to develop a feasible and optimal sequence of operations for a part based upon the technical requirements, including part specifications in the design, the given manufacturing resources, and certain objectives related to cost or time. The task of operation sequencing in CAPP is sequenced by a number of choices in the operation or machine selection, as quoted above. Thus, a combination of different choices and constraints makes process planning a combinatorial problem [18].

2.2. Precedence Cost Matrix (PCM). The precedence cost matrix (PCM) is generated for any pair of features based on the appropriate relative costs involved in each attribute. These costs reflect various parameters such as tool change, machine change, datum, location, and precondition. Generation of variable feasible sequence incorporating the precedence relations makes the problem NP-Complete. An efficient search heuristic will be highly suitable for attempting such problem. In this context, a superhybrid heuristic search named S-GENSAT is preferred for finding the global optimal feasible solution.

\section{State-of-the-Art}

A feasible sequence is deemed to be one which does not violate any of the feasibility constraints (Location reference, Accessibility, Nondestruction, Geometric-tolerance, Strict precedence). Application of the feasibility constraints provide the system with the capability to define a set of precedences between the features of a part resulting in the construction of a feature precedence graph (FPG) to represent these precedence relationships. 
TABLE 3: CAD database for component in Figure 2 [7].

\begin{tabular}{|c|c|c|c|c|c|c|c|c|}
\hline $\begin{array}{l}\text { Feature } \\
\text { no. }\end{array}$ & Feature type & $\mathrm{dx}(\mathrm{mm})$ & dy $(\mathrm{mm})$ & $\begin{array}{c}\text { Width or } \\
\text { radius }(\mathrm{mm})\end{array}$ & $\begin{array}{c}\begin{array}{c}\text { Length } \\
(\mathrm{mm})\end{array} \\
\end{array}$ & $\begin{array}{l}\text { Depth } \\
(\mathrm{mm})\end{array}$ & $\begin{array}{c}\text { Projected } \\
\text { area }\left(\mathrm{mm}^{2}\right)\end{array}$ & $\begin{array}{c}\text { Volume } \\
\left(\mathrm{mm}^{3}\right)\end{array}$ \\
\hline $\mathrm{F} 1$ & Rectangular Pocket & 50 & 90 & 45 & 30 & 20 & 1350 & 27000 \\
\hline $\mathrm{F} 2$ & Thru Hole & 35 & 70 & 7.5 & - & 25 & 176.71 & 4418 \\
\hline F3 & Blind step & 110 & 110 & 20 & 30 & 25 & 600 & 15000 \\
\hline $\mathrm{F} 4$ & Thru hole & 110 & 120 & 6 & - & 35 & 113.1 & 3960 \\
\hline F5 & Thru hole & 30 & 120 & 6 & - & 35 & 113.1 & 3960 \\
\hline F6 & Edge cut & 110 & 0 & 40 & 40 & 30 & 1600 & 48000 \\
\hline F7 & Blind hole & 125 & 15 & 5 & - & 25 & 78.54 & 1960 \\
\hline F8 & Blind step & 30 & 110 & 20 & 30 & 25 & 600 & 15000 \\
\hline F9 & Thru slot & 0 & 60 & 20 & 140 & 25 & 2800 & 70000 \\
\hline F10 & Blind slot & 45 & 0 & 35 & 60 & 25 & 2100 & 52500 \\
\hline
\end{tabular}

TABLE 4: REPMAX for component in Figure 2 [7].

\begin{tabular}{lcccccccccc}
\hline FN & F1 & F2 & F3 & F4 & F5 & F6 & F7 & F8 & F9 & F10 \\
\hline F1 & $\infty$ & 5 & 35 & 25 & 25 & 5 & 5 & 35 & 45 & 5 \\
F2 & 5 & $\infty$ & 5 & 5 & 5 & 5 & 5 & 5 & -45 & 5 \\
F3 & -25 & 5 & $\infty$ & 85 & 5 & 5 & 5 & 5 & 5 & 5 \\
F4 & -15 & 5 & -75 & $\infty$ & 5 & 5 & 5 & 5 & 5 & 5 \\
F5 & -15 & 5 & 5 & 5 & $\infty$ & 5 & 5 & -75 & 5 & 5 \\
F6 & 5 & 5 & 5 & 5 & 5 & $\infty$ & 105 & 5 & 5 & 5 \\
F7 & 5 & 5 & 5 & 5 & 5 & -95 & $\infty$ & 5 & 5 \\
F8 & -25 & 5 & 5 & 5 & 85 & 5 & 5 & $\infty$ & 5 \\
F9 & -20 & 55 & 5 & 5 & 5 & 5 & 5 & 5 & 5 \\
F10 & 5 & 5 & 5 & 5 & 5 & 5 & 5 & 5 & -15 & \\
\hline
\end{tabular}

TABle 5: Cost matrix for case study 2.

\begin{tabular}{lccccccccccccccc}
\hline Operation & 1 & 2 & 3 & 4 & 5 & 6 & 7 & 8 & 9 & 10 & 11 & 12 & 13 \\
\hline 1 & - & 100 & 150 & 100 & 150 & 150 & 150 & 150 & 150 & 650 & 650 & 550 & 550 \\
2 & 100 & - & 50 & 0 & 50 & 50 & 50 & 50 & 50 & 650 & 650 & 650 & 650 \\
3 & 150 & 50 & - & 50 & 0 & 50 & 50 & 50 & 50 & 650 & 650 & 650 & 650 \\
4 & 100 & 0 & 50 & - & 50 & 50 & 50 & 50 & 50 & 650 & 650 & 650 & 650 \\
5 & 150 & 50 & 0 & 50 & - & 50 & 50 & 50 & 50 & 650 & 650 & 650 & 650 \\
6 & 150 & 50 & 50 & 50 & 50 & - & 50 & 50 & 50 & 650 & 650 & 650 & 650 \\
7 & 150 & 50 & 50 & 50 & 50 & 50 & - & 50 & 50 & 650 & 650 & 650 & 650 \\
8 & 150 & 50 & 50 & 50 & 50 & 50 & 50 & - & 50 & 650 & 650 & 650 & 650 \\
9 & 150 & 50 & 50 & 50 & 50 & 50 & 50 & 50 & - & 650 & 650 & 650 & 650 \\
10 & 650 & 650 & 650 & 650 & 650 & 650 & 650 & 650 & 650 & - & 50 & 100 & 150 \\
11 & 650 & 650 & 650 & 650 & 650 & 650 & 650 & 650 & 650 & 50 & - & 150 & 150 \\
12 & 650 & 650 & 650 & 650 & 650 & 650 & 650 & 650 & 650 & 100 & 150 & - \\
13 & 550 & 650 & 650 & 650 & 650 & 650 & 650 & 650 & 650 & 150 & 150 & 50 & - \\
\hline
\end{tabular}

TABle 6: Case study 1.

\begin{tabular}{|c|c|c|c|c|c|}
\hline & Weil et al. [1] & $\begin{array}{c}\text { Bhaskara Reddy } \\
\text { et al. [6] }\end{array}$ & $\begin{array}{c}\text { Krishna and } \\
\text { Rao [12] }\end{array}$ & $\begin{array}{c}\text { Nallakumarasamy } \\
\text { et al. [15] }\end{array}$ & S-GENSAT \\
\hline Optimal cost & 15 & 15 & 15 & 15 & 15 \\
\hline Computational time & - & 30 & 11 & 1 & $<1$ \\
\hline
\end{tabular}


TABle 7: Case study 2.

\begin{tabular}{lcc}
\hline & Turkey et al. [7] & S-GENSAT \\
\hline Optimal cost & -315 & -315 \\
Computational time & - & $6 \mathrm{sec}$ \\
\hline
\end{tabular}

TABle 8: Case study 3.

\begin{tabular}{lccc}
\hline & GA & SAT & S-GENSAT \\
\hline Optimal cost & 1350 & 1300 & 1200 \\
Computational time & 15 & 13 & 7 \\
\hline
\end{tabular}

Let us consider a part composed of $\mathrm{N}$ features which can be processed by $\mathrm{N}$ factorial sequences. However, many sequences turn out to be an infeasible one, as it violates the precedence constraints; very few attempts have been made for determining the optimal sequence incorporating precedence relations which is vital for reduction of solution space. In the present study, the operation sequencing problem has been attempted by a superhybrid technique, SGENSAT, a hybrid metaheuristic. A brief introduction to SGENSAT and solution space reduction is described in the following sections for better understanding of the proposed methodology.

\section{Superhybrid GENSAT}

Genetic Algorithms (GAs) are adaptive heuristic search algorithms premised on the evolutionary ideas of natural selection and genetics. The basic perception of GA is designed to simulate processes in a natural system necessary for evolution, specifically to those that follow the principles laid by Charles Darwin of survival of the fittest. Simulated annealing technique (SAT) is an enhanced version of global search. Annealing refers to the process when physical substances are raised to a high-energy level and then gradually cooled until some solid state is reached. The goal of this process is to reach the lowest energy state. In this process physical substances usually move from higher energy states to lower ones if the cooling process is sufficiently slow. However, there is some probability at each stage of the cooling process that a transition to a higher energy state will occur, but this probability of moving to higher energy state decreases in this process. As GA and SAT are extensively discussed in the literature, the focus of this paper is converged towards hybridization and solution space reduction technique (SSRT).

4.1. Hybridization of GA and SAT. As mentioned in the introduction section, Evolutionary algorithms, which mimic living organisms in achieving optimal survival solutions, can often outperform conventional optimization methods. In the past two decades, GA has been widely applied for solving complex manufacturing problems. GA operates on the whole population, and the search process may be trapped in local optima, while SA possess the capability of jumping out of local minima and proceeds towards global minima.
The proposed schema is divided into two phases: the GA and SAT. During the first phase, GA generates an initial population randomly incorporating SSRT. Then, cross-over and mutation operators are imposed for offspring generation based on the initial population. The cross-over and mutation sites are selected randomly. This process is repeated for a period of generations for attaining an optimum solution. Then, the GA sends the optimal solution for SAT (PhaseII) for investigation of further improvement. This hybrid technique improves the solution quality and reduces the computational time reasonably.

4.2. Solution Space Reduction Technique (SSRT). To demonstrate the importance of SSRT, a case study is considered from [6], as shown in Figure 1. The operations to be performed on the component are mentioned as A1, B1, B2, C1, D1, D2, D3, and E1. The technical specifications, dimensions, and geometrical tolerances are also indicated. Based on the precedence graph, a precedence cost matrix is generated for each pair of features by taking into consideration of various attributes like machining parameter change, cutting tool change, set-up change, and machine tool change, and is given in Table 1. The symbol "- " indicates an invalid task. The operation precedence graph consisting of feasibility constrains are shown in Figure 2.

As the component comprises of 8 operations, the total possible sequence of operations are 40320 . However, after incorporation of precedence constrains, the feasible sequence of operations are greatly reduced to 10 as illustrated in Figure 3.

Whereas, Bhaskara Reddy [6] generated sequences in the initial population which violates the precedence relations even though the final reported optimal solution is a feasible one for the same case study. As the total number of feasible sequence is reduced to 10 , even a search technique is not essential to find the optimal solution. The optimal sequence is the same as reported by [6] as illustrated in Table 2 . However, the solution space reduction is $99.975 \%$. Hence, the proposed work suggests to incorporate the precedence constrains for generation of any sequence at any stage of the program, which greatly reduces the computational time and it easily tends to global optima.

\section{Case Study 1}

To illustrate the proposed methodology, a machine component is considered from [7], as shown in Figure 4. The $\mathrm{CAD}$ data base and the corresponding reward penalty matrix (REPMAX) for the sample component are listed in Tables 3 and 4 , respectively.

\section{Case Study 2}

To test the robustness of the algorithm, another case study of an industrial component is considered. This component is a part with 13 operations done on a machining center. The cost matrix for the newly proposed component is given in Table 5 . 


\section{Results and Discussion}

The proposed optimization of CAPP based on Superhybrid Algorithm developed in this work has been applied on three different case studies. The robustness of proposed algorithm and obtained results were compared with the previous works and are listed in the following tables. This S-GENSAT is coded in Turbo $\mathrm{C}++$ on Pentium core 2 Duo computer with a $2.4 \mathrm{GHz}$ processor.

Optimal solution for case study mentioned in Section 4.2 is listed in Table 6 and compared with previous works. The optimal solution is the same as reported in the literature; however, using SSRT the computational time greatly reduced as well as the possible feasible sequences are reduced to 10 rather than searching the whole solution space of 40,320. Table 7 exhibits the output parameters for case study 1 . The optimal cost is found to be -315 units (open ended fitness value), and the computational time taken for execution is only $6 \mathrm{sec}$. Results for case study 2 are elaborated in Table 8. The proposed algorithm is compared with a conventional Genetic algorithm and simulated annealing technique. Present results clearly reveal that S-GENSAT is superior to other optimization techniques.

\section{Conclusion}

In this paper, we propose Superhybrid Algorithm S-GENSAT, a hybrid metaheuristic for solving operation sequencing problem in CAPP. The proposed methodology demonstrated a high rate of success, being able to solve problems for which analytical and other heuristic optimization routines fail. From the results obtained, it is clear that S-GENSAT produces improved optimal sequences with a lesser computational time. In a CAPP system, an efficient heuristic search is required to explore the large solution space of valid operational sequences under various interacting constraints. This paper deals with Hybrid S-GENSAT algorithm which incorporates Solution space reduction technique (SSRT) for solving NP-Complete combinatorial problem, to get more alternate optimal sequence in a single run with lesser computational time. It seems that the algorithm can be treated as quite effective as in most cases and it finds a solution which represents a good approximation to the optimal one and fast enough (the number of iterations and running time of the algorithm are reasonable). The result shows that the algorithm is effective in solving the NP-complete problems like operation sequencing. Also, proposed algorithm is a very robust one that is able to search wide areas of the solution space, escape from local minima, and is guaranteed to converge with a lesser computation time.

\section{References}

[1] R. Weill, G. Spur, and W. Eversheim, "Survey of computeraided process planning systems," Annals of CIRP, vol. 31, no. 2, pp. 539-551, 1982.

[2] C. J. Lin and H. P. Wang, "Optimal operation planning and sequencing: minimization of tool change overs," International Journal of Production Research, vol. 31, no. 2, pp. 311-324, 1993.
[3] C. Koulamas, "Operations sequencing and machining economics," International Journal of Production Research, vol. 31, no. 4, pp. 957-975, 1993.

[4] S. A. Irani, H. Y. Koo, and S. Ranam, "Feature based operation sequence generation in CAPP," Production Research, vol. 33, pp. 17-39, 1995.

[5] J. M. Usher and R. O. Bowden, "The application of genetic algorithms to operation sequencing for use in computer-aided process planning," Computers and Industrial Engineering, vol. 30, no. 4, pp. 999-1013, 1996.

[6] S. V. Bhaskara Reddy, M. S. Shunmugam, and T. T. Narendran, "Operation sequencing in CAPP using genetic algorithms," International Journal of Production Research, vol. 37, no. 5, pp. 1063-1074, 1999.

[7] T. Dereli and I. H. Filiz, "Optimisation of process planning functions by genetic algorithms," Computers and Industrial Engineering, vol. 36, no. 2, pp. 281-308, 1999.

[8] D. H. Lee, D. Kiritsis, and P. Xirouchakis, "Branch and fathoming algorithms for operation sequencing in process planning," International Journal of Production Research, vol. 39, no. 8, pp. 1649-1669, 2001.

[9] F. Zhang, Y. F. Zhang, and A. Y. C. Nee, "Using genetic algorithms in process planning for job shop machining," IEEE Transactions on Evolutionary Computation, vol. 1, no. 4, pp. 278-289, 1997.

[10] H. Foerster and G. Wäscher, "Simulated annealing for order spread minimization in sequencing cutting patterns," European Journal of Operational Research, vol. 110, no. 2, pp. 272281, 1998.

[11] W. D. Li, S. K. Ong, and A. Y. C. Nee, "Optimization of process plans using a constraint-based tabu search approach," International Journal of Production Research, vol. 42, no. 10, pp. 1955-1985, 2004.

[12] A. G. Krishna and K. M. Rao, "Optimisation of operations sequence in CAPP using an ant colony algorithm," Advanced Manufacturing Technology, vol. 29, no. 1-2, pp. 159-164, 2006.

[13] Y. W. Guo, A. R. Mileham, G. W. Owen, and W. D. Li, "Operation sequencing optimization using a particle swarm optimization approach," Proceedings of the Institution of Mechanical Engineers, Part B: Journal of Engineering Manufacture, vol. 220, no. 12, pp. 1945-1958, 2006.

[14] M. Salehi and R. Tavakkoli-Moghaddam, "Application of genetic algorithm to computer-aided process planning in preliminary and detailed planning," Engineering Applications of Artificial Intelligence, vol. 22, no. 8, pp. 1179-1187, 2009.

[15] G. Nallakumarasamy, P. S. S. Srinivasan, K. Venkatesh Raja, and R. Malayalamurthi, "Optimization of operation sequencing in CAPP using simulated annealing technique (SAT)," International Journal of Advanced Manufacturing Technology, vol. 54, no. 5-8, pp. 721-728, 2011.

[16] Y. F. Wang, Y. F. Zhang, and J. Y. H. Fuh, "Using hybrid particle swarm optimization for process planning problem," in Proceedings of the International Joint Conference on Computational Sciences and Optimization (CSO '09), pp. 304-308, Sanya, China, April 2009.

[17] K. Ganesh and M. Punniyamoorthy, "Optimization of continuous - time production planning using hybrid genetic algorithms_-simulated annealing," The International Journal of Advanced Manufacturing Technology, vol. 26, no. 1-2, pp. 148-154, 2005.

[18] U. P. Korde, B. C. Bora, K. A. Stelson, and D. R. Riley, "Computer-aided process planning for turned parts using fundamental and heuristic principles," Journal of Engineering for Industry, vol. 114, no. 1, pp. 31-40, 1992. 

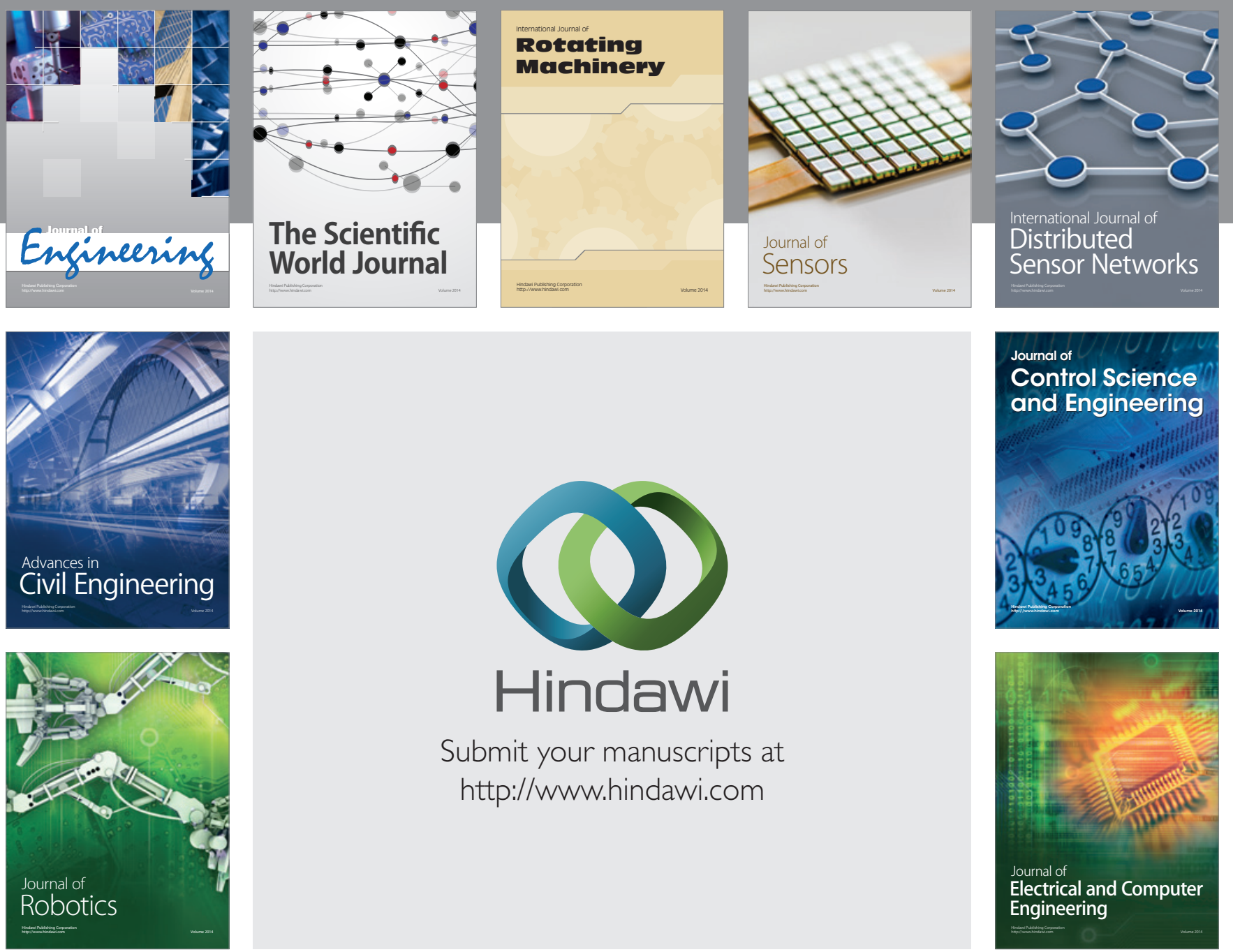

Submit your manuscripts at

http://www.hindawi.com
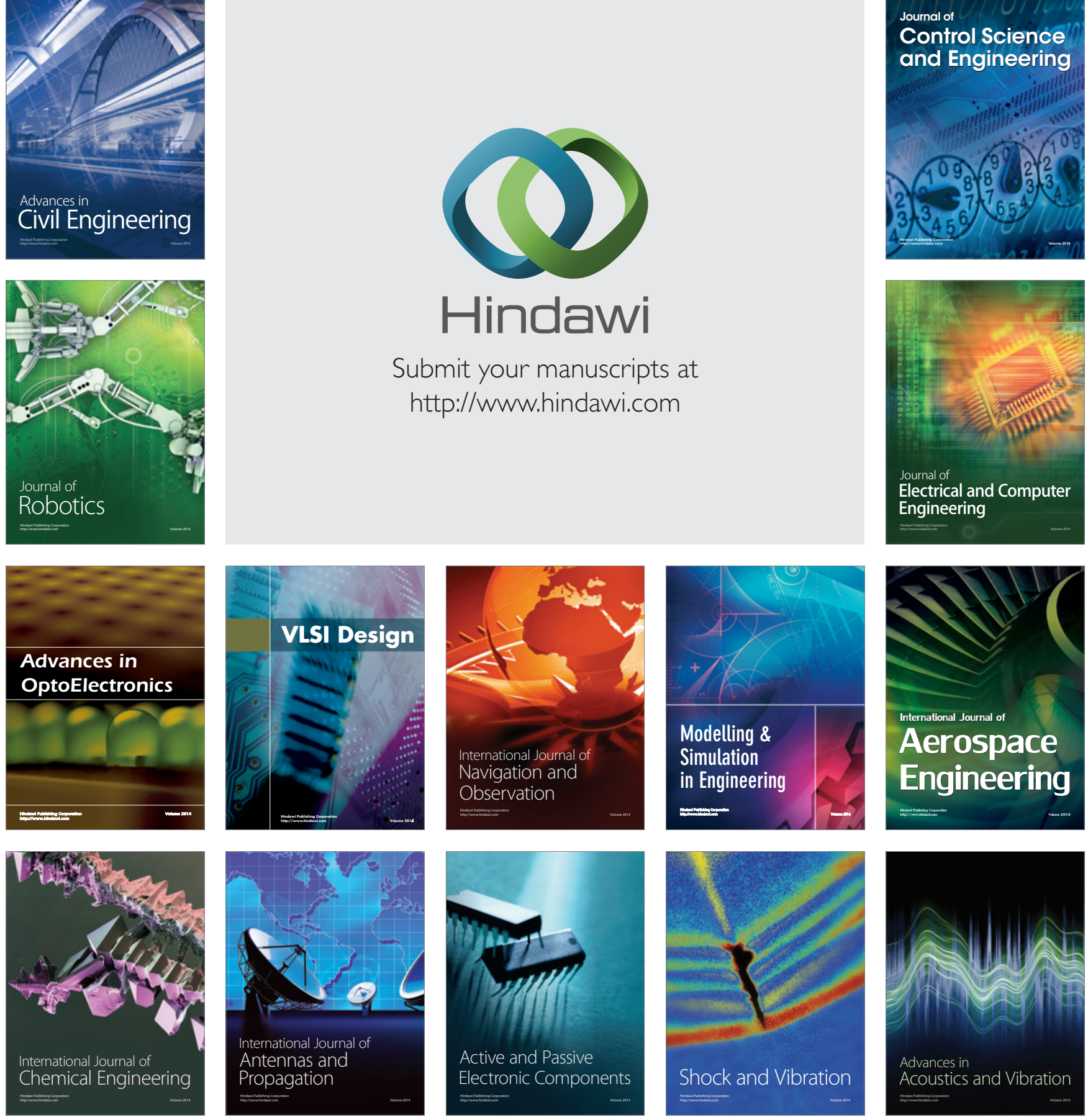